The International Journal of Multimedia \& Its Applications (IJMA) Vol.3, No.3, August 2011

\title{
APPROACH GEOMETRY/IMAGE FOR RENDERING FOREST IN REAL TIME
}

\author{
ABBAS FAYÇAL ${ }^{1}$ and Med CHAOUKI BABAHENINI ${ }^{2}$ \\ ${ }^{1}$ Department of Computer, Mohamed Khider University, BISKRA, ALGERIA \\ abbas.faycal@gmail.com \\ ${ }^{2}$ Department of Computer, Mohamed Khider University, BISKRA, ALGERIA \\ Chaouki.babahenini@gmail.com
}

\begin{abstract}
Representation and visualization of a forest in real time is a difficult task, the main difficulty of this problem is the complexity, forest scenes contain a very large quantity of small details difficult to model, expensive to make and require very large amount of memory. The goal of our research is to present an approach for modeling and rendering of natural objects that must be used to bypass the obstacle of the geometric complexity and provide simple model for the creation of natural structures. Our work focuses on trees that represent the forest. To simplify the complexity of the scene, the trees from a distance are represented by billboards (single, cross, ...) for tree views near, we propose an hybrid model between billboards and L-systems, where we separate the tree into two separate trunks and branches are represented by an L-system grammar and leaves are represented by billboards, this increases the realism of nearly trees from the point of view.
\end{abstract}

\section{KEYWORDS}

Image-based rendering, Natural scenes, Real-time rendering, L-system, Billboards.

\section{INTRODUCTION}

Since more than 25 years ago, computer graphics research has taken special attention to the visual representation of natural scene, many of the current interactive applications such as flight simulators, virtual reality environments or computer games take place in outdoor scenes.

One of the essential components in these scenes is trees, the interactive rendering of vast natural scene, involving hundreds of thousands of trees and other vegetal species is an open problem. One of the major challenges is that the large number of polygons needed to model a tree or a forest exceeds the limits posed by the current rendering hardware [1].

Rendering forest scenes in real time with traditional modeling methods is a difficult task; the main obstacle of this complexity comes from geometry. Among the approaches that emerged to circumvent the obstacle of the geometric complexity, the image-based rendering [2]. The principle of this approach is to use the image as a primitive rendering, it uses a set of images taken at different points of view to reconstruct the final scene, the main interest of this technique is that the computation time is independent of the geometric complexity of the scene the trees from a distance are represented by billboards (single, cross, ...) for tree views near, we propose an hybrid model between billboards and L-systems, where we separate the tree into two separate 
The International Journal of Multimedia \& Its Applications (IJMA) Vol.3, No.3, August 2011

trunks and branches are represented by an L-system grammar and leaves are represented by billboards, this increases the realism of nearly trees from the point of view.

\section{PreVious WORK}

The image-based rendering system proposed by Jakulin [3] introduced a simplified billboard based rendering approach that instead of dynamicallycalculated slices, uses multiple asix-aligned slicing to capture the foliage into a texture, and combines these with a geometric base. His method results in nice results by only using 3-7 slices, which equals 6-14 billboards.

Meyer et al. [4] provides a framework for rendering trees with complex effects such as shading, self shadowing, and dynamic illumination. They combine a hierarchy of bidirectional textures (HBT) to provide billboards for each given observer and light directions with a hierarchical visibility structure for self-shadows and cast shadows. This representation is efficient for trees, as it is hierarchical display lists calls are heavily used, This approach requires interpolation of several images at rendering time, and the method is only addressed to far views of large real-time forest.

Neyret converts complex natural objects into volumetric textures, which are then raytraced [5]. Mip-mapping is used to reduce texture data, and to generate samples from trees in the distance. The method provides high quality images of very complex scenes at moderate rendering times of several minutes, but it is not applicable in interactive applications. More recently, this technique was adapted to graphics hardware by Decaudin and Neyret [6]. The results are very effective for far views of large natural scenes but lacks from a LOD technique to deal with a smooth transition in closer views.

The "billboard clouds" approach : introduced by Decoret et al. [7], represent an object by one or more intersecting textured quads. They share the advantages of image based representation, such as low rendering cost and MIPmap filtering with the distance. However, the correct handling of semi-transparent billboard regions has not been addressed satisfactorily. Even if the original object is opaque, its contours will become semi-transparent with MIP-map filtering. Sorting billboards for correct transparency is costly and requires splitting the (numerous) billboards which intersect each other. Furthermore, if several such intersecting objects have to be considered, the sorting and splitting must be done globally (at runtime if objects move).

\section{OUR APPROACH}

We propose a hybrid geometry-image representation for the real-time rendering of realistic forests that used to rendering many trees with a low cost. The system is responsible for producing synthetic images of a forest, our system has two types of representations, for trees in the backgrounds that are represented by the technique of billboard and because the unrealistic perfect for trees represented by this technique, we propose an hybrid model between billboards and Lsystems, where we separate the tree into two separate trunks and branches are represented by an L-system grammar and leaves are represented by billboards, this increases the realism of nearly trees from the point of view.

\subsection{Billboarding}

Billboards are the most common tool for real time rendering of forests. Thanks to their low cost, they are still considered the best choice in many recent industrial simulators. They are used in two different ways: classical billboards are small images that always face the camera [8]. 
To implement billboard it requires the modelview matrix, this matrix contain all the geometrical transformations, required to change your input coordinates into camera coordinates.

We will need to know the camera up vector and position and the billboard position. We will use these to calculate the local billboard's axis. The billboard look vector is calculated from the camera position and billboard position. The vector is from the billboard to the camera;

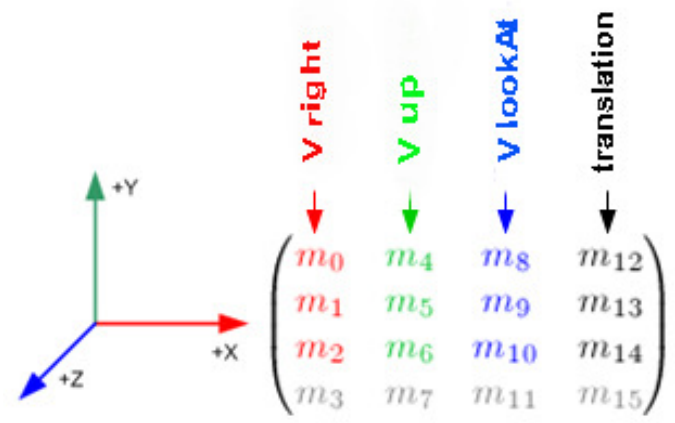

Figure 1. Extraction Information

The submatrix consists of 3 columns: the first is the right vector, the second is the up vector and the third one is the lookAt vector. In case we wanted to use the billboard for trees, grass as discussed earlier, we do not want the billboard to be spherical but instead cylindrical. If this is what we want we do not touch up vector stay in the submatrix.
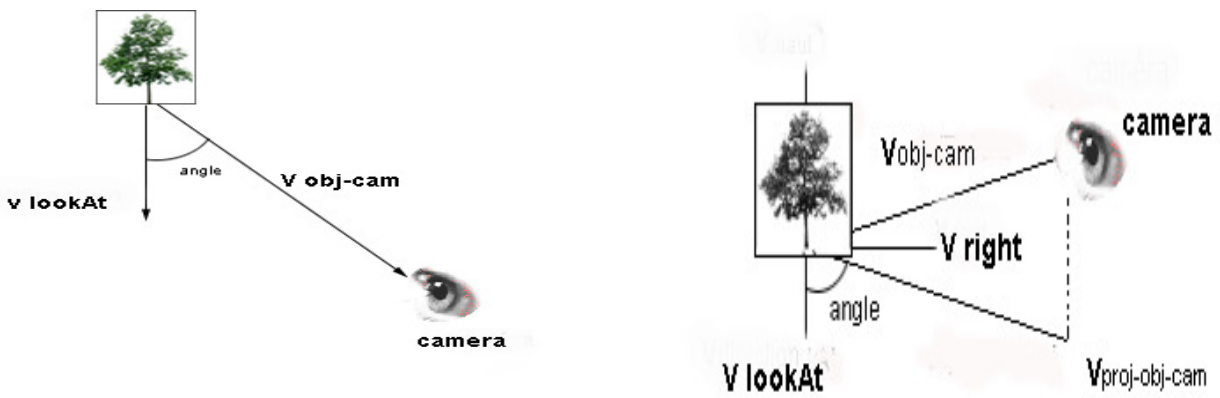

Figure 2. Billboard representation from the camera.

For simplicity reasons it is assumed that the object has the following vectors:

right vector $=\{1,0,0\}$, up vector $=\{0,1,0\}$, lookAt vector $=\{0,0,1\}$

In practice this means that the object is drawn in the local origin, and it is looking along the positive $\mathrm{Z}$ axis. In order to orientate the object a rotation is performed around the up vector, by the angle between the lookAt vector and the vector from the object to the camera.

The vector from the object to the camera can be defined as objToCam $=$ CamPos - ObjPos. The vector objToCamProj is the projection of objToCam in the $\mathrm{XZ}$ plane. Therefore its $\mathrm{Y}$ component should be set to zero. If objToCamProj is normalized then the inner product between lookAt and objToCam will allow the computation of the cosine of the angle. However knowing the cosine alone is not enough, since the $\cos (\alpha)=\cos (-\alpha)$. Computing the cross product as well allows us to uniquely determine the angle. The cross product vector will have the same direction as the up 
The International Journal of Multimedia \& Its Applications (IJMA) Vol.3, No.3, August 2011

vector if the angle is positive. For negative angles the up vector's direction will opposed to the up vector, effectively reversing the rotation.

\subsection{L-system}

L-Systems [9] are developed as a means of describing the development of cellular organisms. Przemyslaw Prusinkiewicz [10] subsequently develops a technique for modelling plants using LSystems. L-Systems are a very compact, biologically based means of modelling the developmental systems of plants.

Our representation inspired from a widely used method for interpretation in the realistic modeling of plants called turtle interpretation. A state of the turtle is defined as a triplet (x, y, a), where the Cartesian coordinates $(\mathrm{x}, \mathrm{y})$ represent the turtle's position, and the angle a, called the heading, is interpreted as the direction in which the turtle is facing.

\section{Generating the structure of the tree}

When drawing tree-like structures, a "turtle state" is maintained. When starting a new branch we save the current position and orientation and then draw the branch. When we are done drawing, we restore the state and are ready for the next operation. This process can be thought of as a stack of states using push and pop operations as the saving and restoring of states. This method is simple and easy to implement when creating $2 \mathrm{D}$ line drawings.
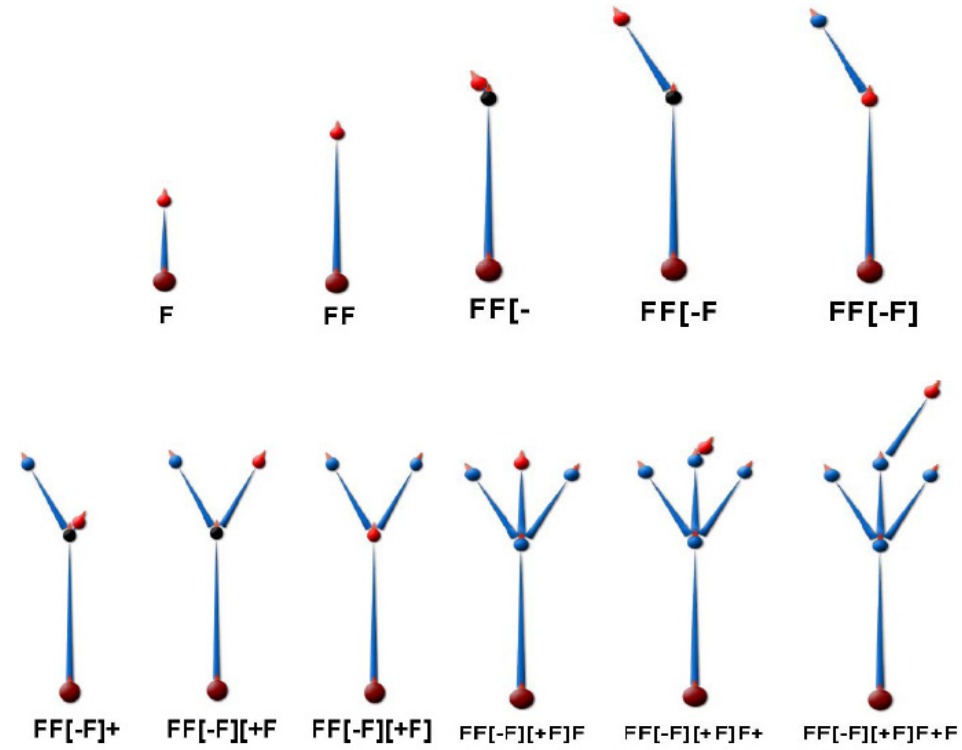

Figure 3. Constructing a skeleton representation

After the creation of words describing the topology of the tree, we represent a three-dimensional tree from a particular word, we need then to give a geometric interpretation to symbols component.

\section{Geometric interpretation}

Based on the simple observation that the branches are more or less cylindrical, The base primitive we used to give volume to each branch segment is the cylinder. Thus, giving a range for each segment of the skeleton of the tree, we were able to obtain results more visually representative than just "wire". 
The International Journal of Multimedia \& Its Applications (IJMA) Vol.3, No.3, August 2011

To ensure connectivity of the object in the end, some information is needed, it is the position of the ends of the segment to avoid a discontinuity at the junction of two cylinders of different radii and information giving the size of the ends the cylinder, we must store the diameter at each end and the radius of the cylinder base.

To define precisely the form of trunk and branches of the tree we must apply a texture on 2D cylindrical.

So far we have treated as branches and the trunk covering the tree structure. However, there is still an essential part of the tree, which provides substantial weight to the visual realism of the tree: leaves.

\section{Generation of the leaves}

Our idea is to insert a character in the alphabet of L-system, the geometric interpretation of this character is a billboard, a semi-transparent rectangle represents small branches and leaves of the tree. A blending operation is required to view these quads semi-transparent. We combine the test with the alpha blending. The alpha test operation removes part completely transparent between the sheets and the edge of the texture. The blending takes care of the pixels composing the edges of the leaves with the background. (Figure 4.) shows a tree obtained by our method.

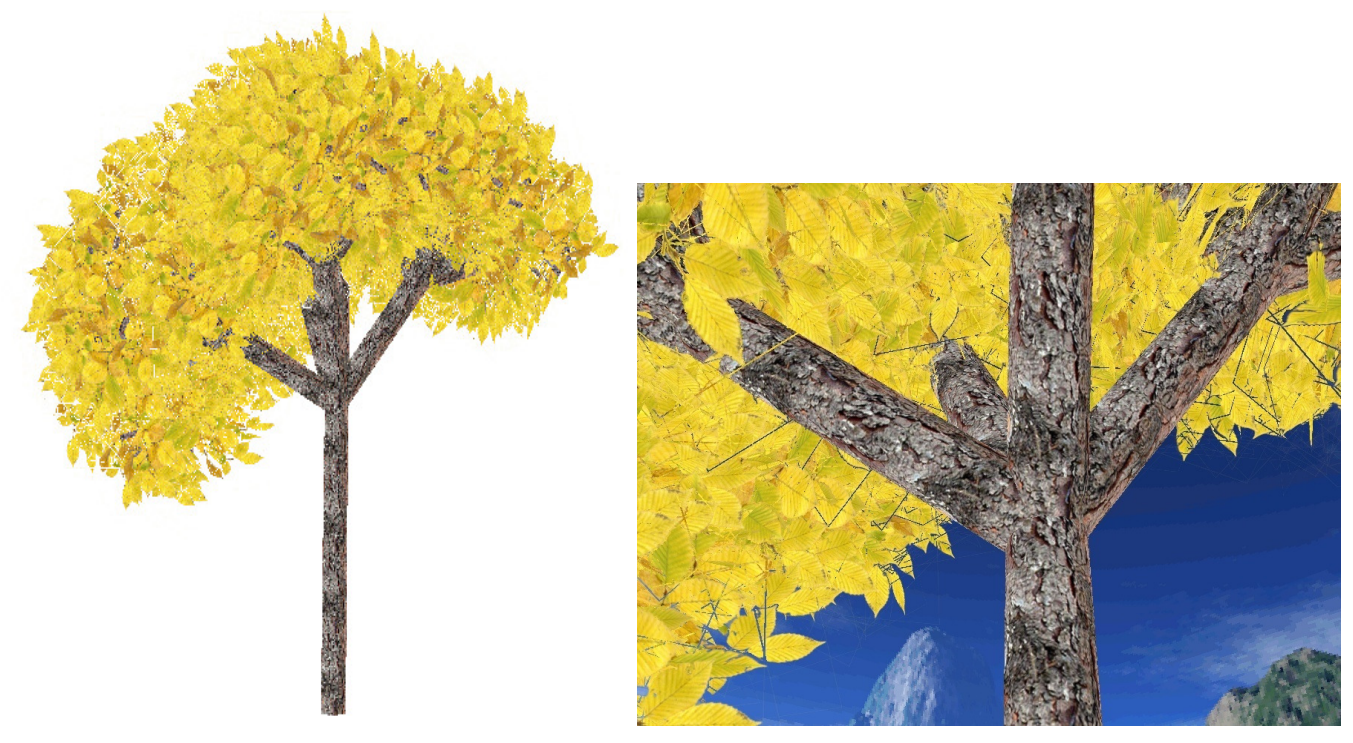

Figure 4. Tree obtained by our method.

\section{Results}

We tested our algorithm with a machine Pentium Core 2 Duo $2.40 \mathrm{GHz}$ with $2.0 \mathrm{~GB}$ of DDR memory and an NVidia GeForce 9400 GT with 512 MB memory.

We present some results showing the variation in computation time by the number of trees and number of billboard. We are changing the number of trees generated which directly affects the computation time consumed:

compares the frequency display for two scenes constructed using the flat billboards and billboards on the cross. 
The International Journal of Multimedia \& Its Applications (IJMA) Vol.3, No.3, August 2011

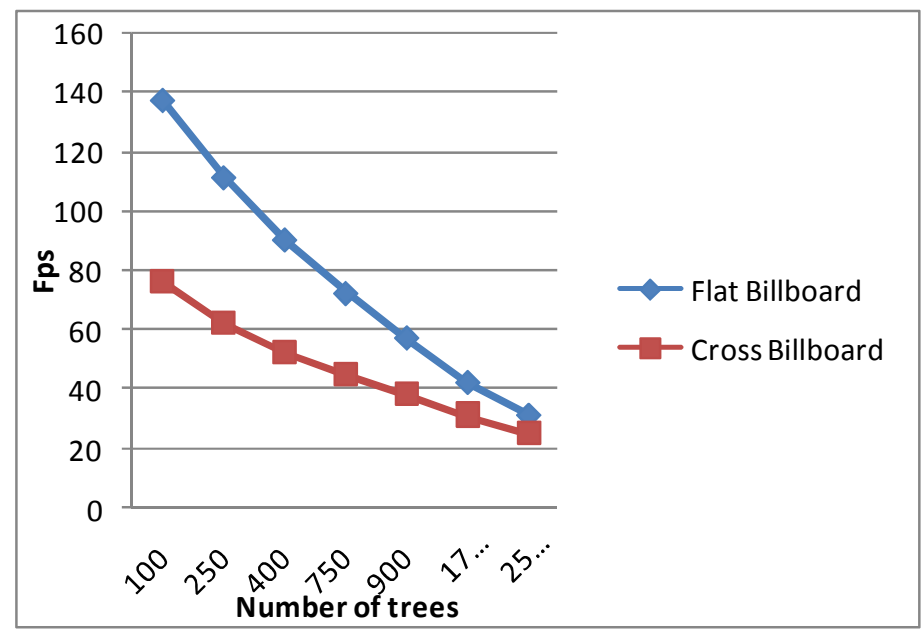

Figure 5. Comparison between a model of flat Billboard and Cross Billboard ( 8 billboard on cross).

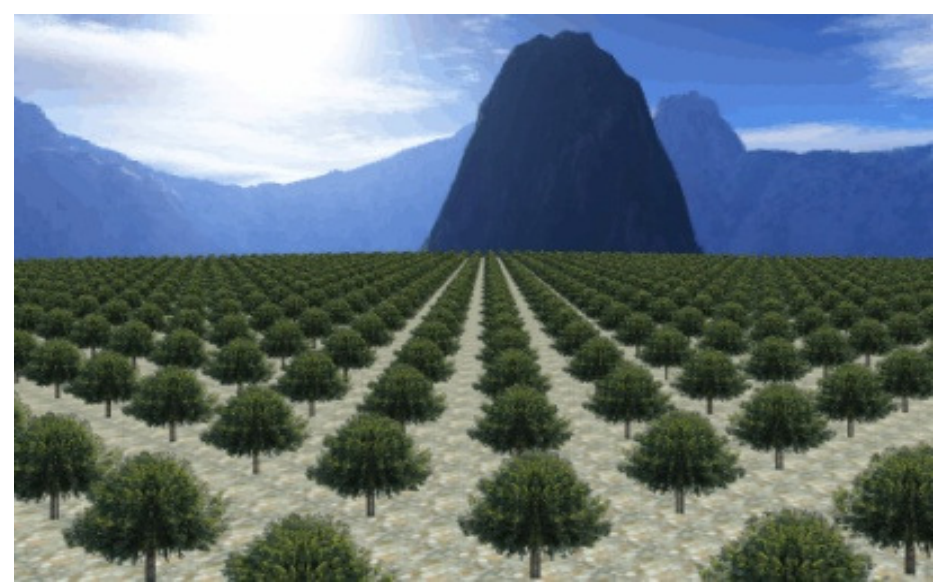

Figure 6. Forest scene with 1600 trees. each tree represented by 8 cross billboard.

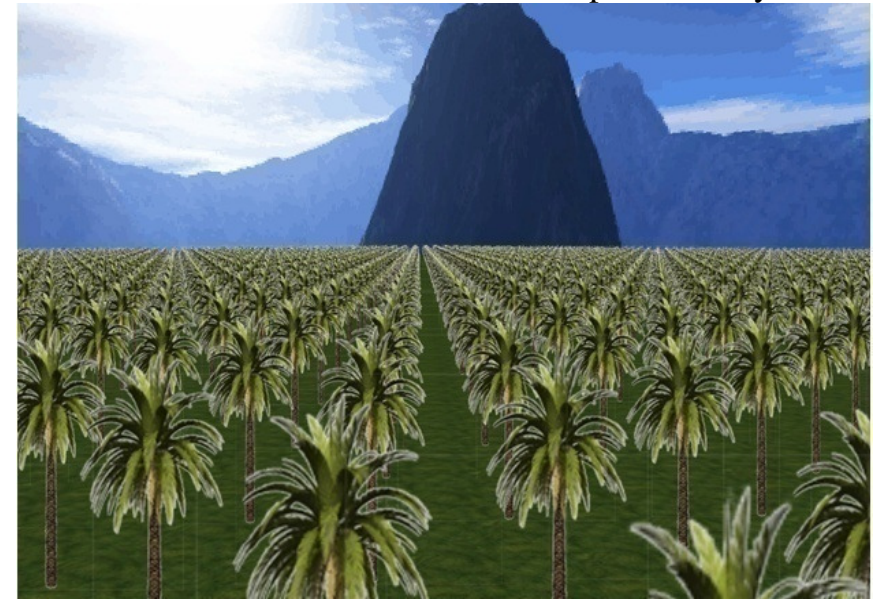

Figure 7. Forest scene with 1600 trees. each tree represented by billboards flat. 
We note that for those scenes, we were able to display 2500 trees at a frequency display 25 frames per second, which brings us back to say that our record is done in real time. In this section we present a hybrid model for the foreground trees, the tree structure is generated by an 1-system and leaves by the billboards.

Figure 8. presents some trees obtained by our method. The trunk is generated by an L-system grammar. The string defining the rules of growthF [+ FL] [FL-] [> FL], where F shows a branch, and $\mathrm{L}$ shows a billboard.

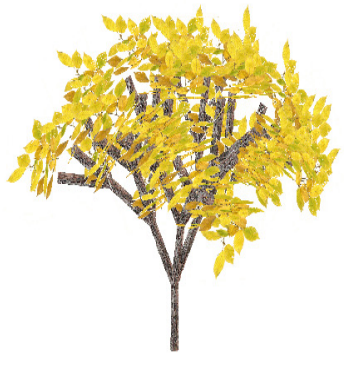

40 billboards

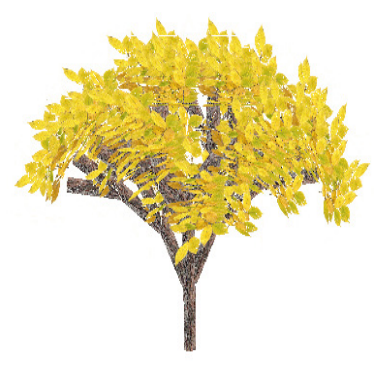

90 billboards

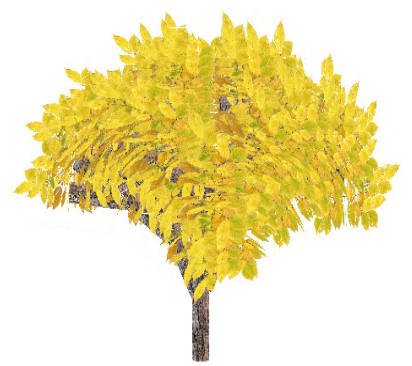

200 billboards

Figure 8. Trees rendered with different number of billboards.

In order to evaluate our model in terms of performance, we make a comparison with a purely geometric model where the bark is generated by an L-system and the leaves are modeled by a polygon with more sides.
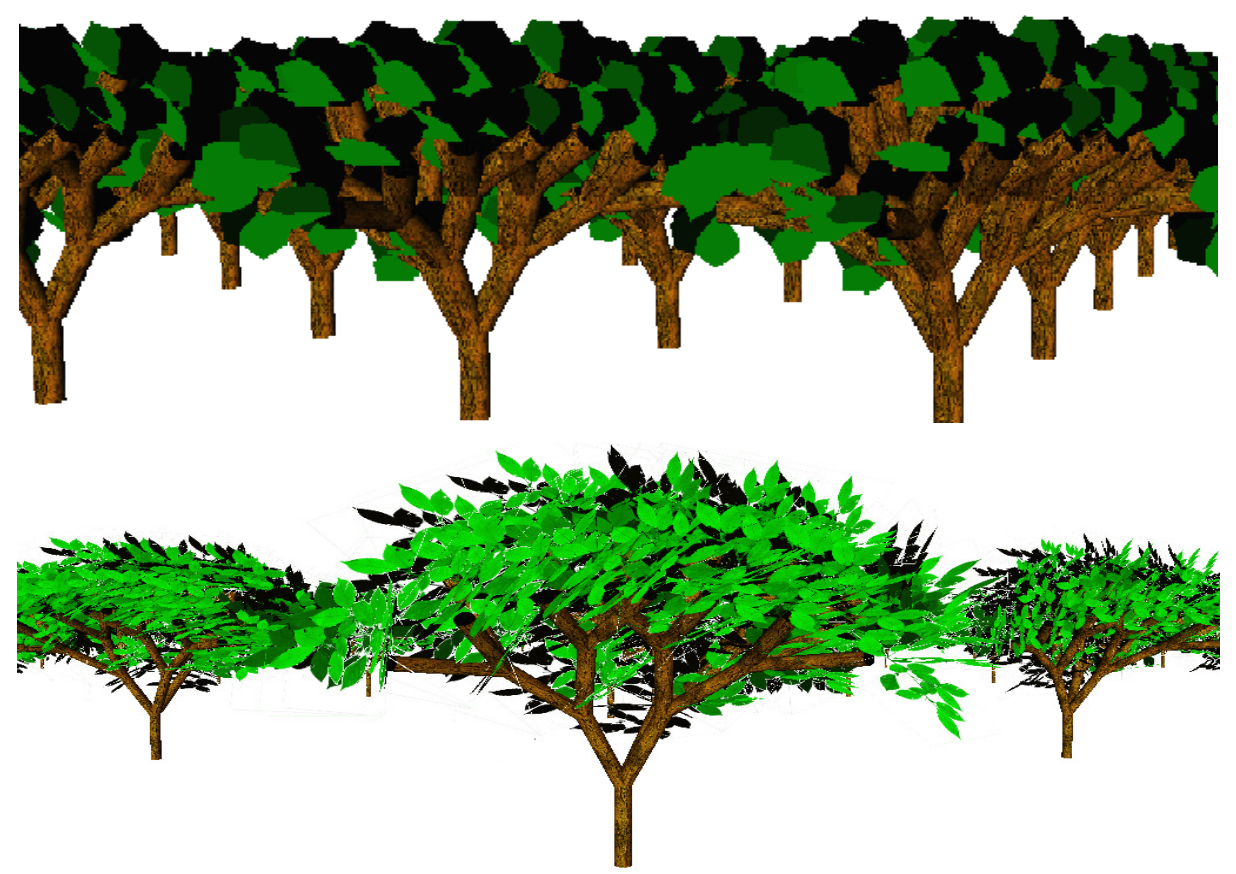
The International Journal of Multimedia \& Its Applications (IJMA) Vol.3, No.3, August 2011

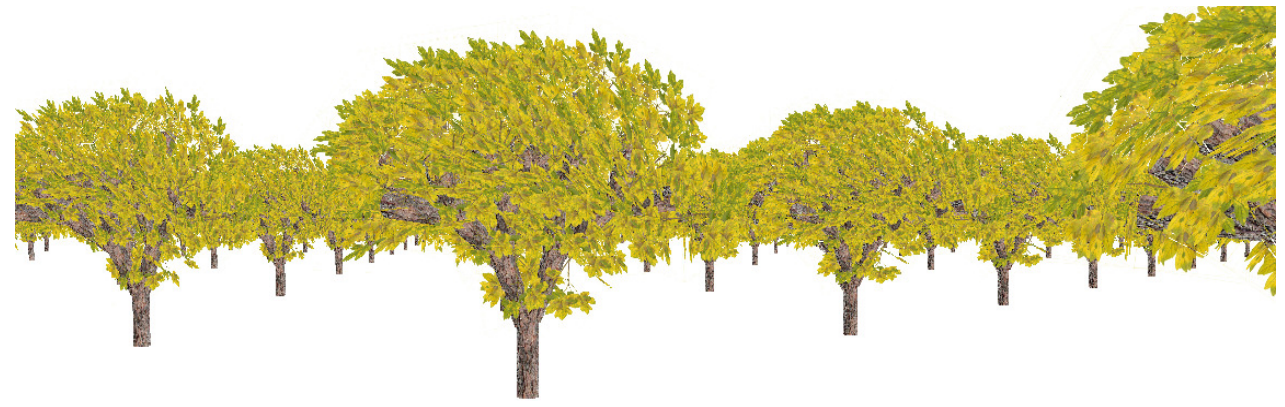

Figure 9. Visual comparisons of the pure geometric model and the hybrid model Geometry / image suggested.

In terms of visual quality, we found that our method increases the realism of the trees with the use of billboards, which are images of real leaves.

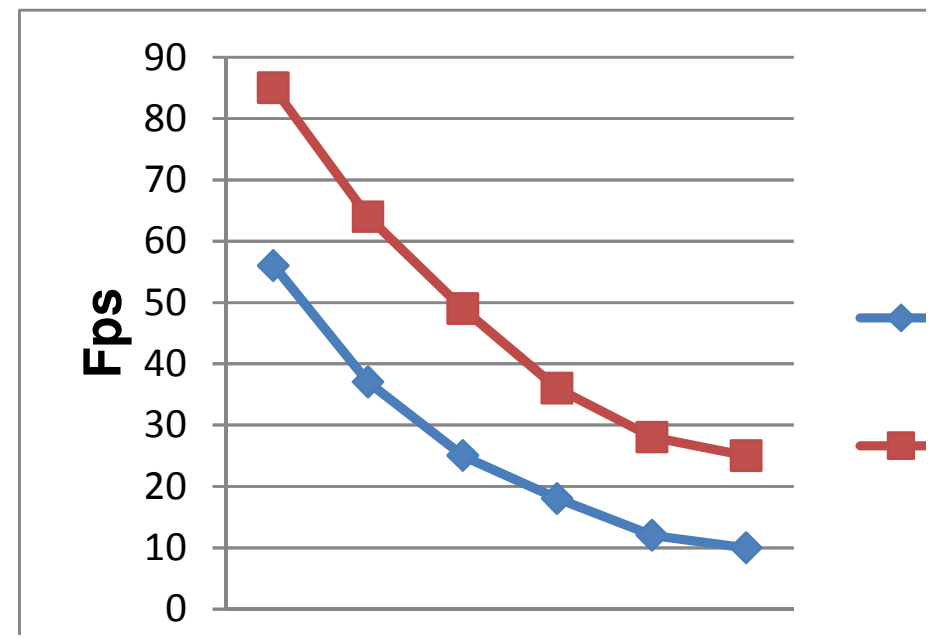

Figure 10. Comparison of the pure geometric model and the hybrid model Geometry / image suggested.

As shown in (Figure 10), our technique that replaces a set of primitives (leaves and branches) in more effective representation has improved the frame rate (in frames per second). It also allowed us to render efficient and rapid improvement of the complexity and visual quality of forest complex scenes.

Finally, we present the results obtained by the method we proposed, in every scene we change the number of trees and textures of leaves and branches. 
The International Journal of Multimedia \& Its Applications (IJMA) Vol.3, No.3, August 2011

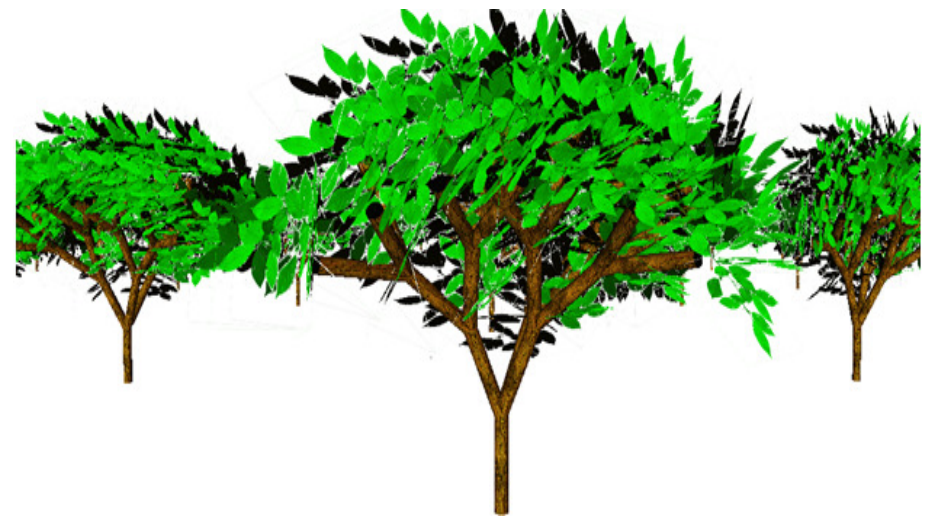

Figure 11. Forest scene with 100 trees with lighting and shading.

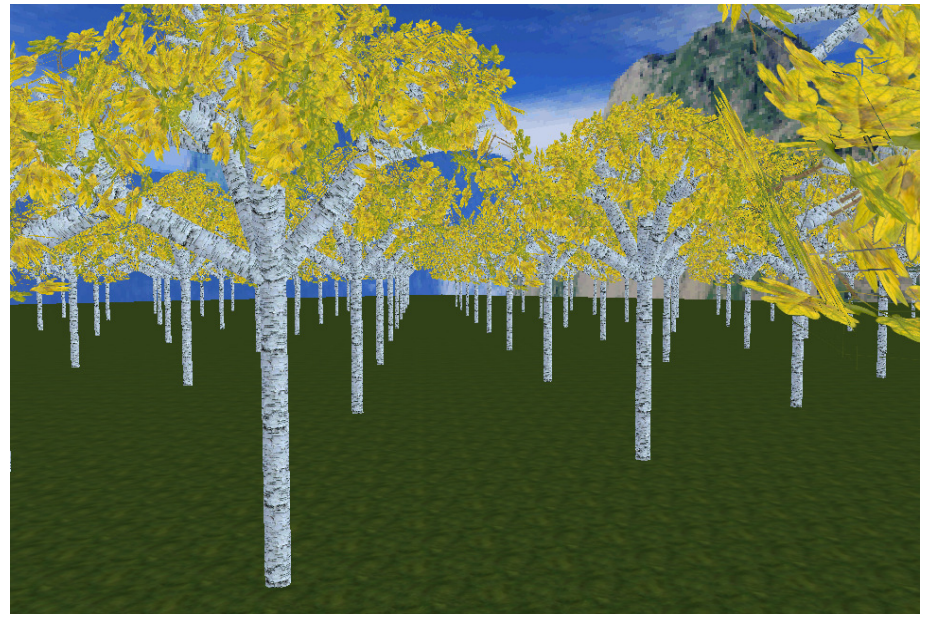

Figure 12. Forest scene with 400 trees.

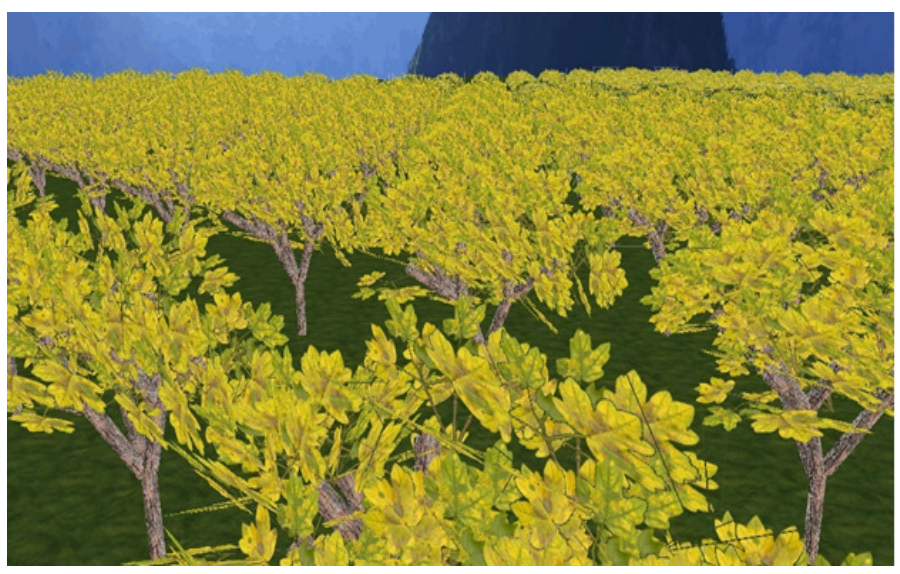

Figure 13. Forest with 520 trees (multi-representation): 120 trees L-System_billboard, 120 trees billboards 8-crossed, 280 trees billboards flat. 
The International Journal of Multimedia \& Its Applications (IJMA) Vol.3, No.3, August 2011

\section{CONCLUSIONS}

We introduced a representation that treat the complexity of a forest by replacing a set of primitives by a representation more effective, this representation allows efficient rendering of trees (quality) and fast (real time). We achieved this goal by implementing a technique of impostors (billboarding) by mixing different types of billboards, and different representation. A hybrid representation for nearly trees from the point of view, and billboards with less detail in trees away from the observer. An hybrid model between billboards and L-systems, where we separate the tree into two separate trunks and branches are represented by an L-system grammar and leaves are represented by billboards, this increases the realism of nearly trees from the point of view.

For future work, we plan to use the maximum computing power with the integration of current graphics cards (GPU) and the parallelization of this algorithm for optimal performance reasons and to allow the central processor (CPU) Free perform other tasks.

\section{REFERENCES}

[1] Oliver Deussen, Pat Hanrahan, Bernd Lintermann, Radomir Mech, Matt Pharr, and Przemyslaw Prusinkiewicz. (1998) "Realistic modeling and rendering of plant ecosystems". In ACM Computer Graphics Proceedings, Annual Conference Series 1998, pp. 275-286.

[2] Chan, S.C. Heung-Yeung Shum, King-To Ng.(2007)," Image-Based Rendering and Synthesis". Signal Processing Magazine, IEEE, Volume: 24 Issue:6, page(s): 22 - 33.

[3] Aleks Jakulin.( 2007), "Interactive Vegetation Rendering with Slicing and Blending". In Proceedings of Eurographics (Short Presentations).

[4] Alexandre Meyer, ( 2001). "Représentations d'arbres réalistes et efficaces pour la synthèse d'image de paysages", Thèse de Doctorat, Université Joseph Fourier.

[5] Fabrice Neyret,( 1998), "Modeling Animating and Rendering Complex Scenes using Volumetric Textures". In IEEE Transactions on Visualization and Computer Graph- ics, volume 4(1).

[6] Philippe Decaudin and Fabrice Neyret , (2009), "Volumetric Billboards",1LJK/IMAG-INRIA Grenoble, France

[7] Xavier Decoret, Frédo Durand, François X. Sillion et Julie Dorsey,(2002). "Billboard Clouds" , Rapport d'activité INRIA.

[8] Siggraph Course Notes CD-ROM. Advanced Graphics Programming Techniques Using OpenGL. Addison-Wesley.

[9] A. Lindenmayer. (1968), "Mathematical models for cellular interaction in development", parts I and II. Journal of Theoretical Biology, 18:280-315.

[10] Przemyslaw Prusinkiewicz, Aristid Lindenmayer, and James Hanan. Developmental models of herbaceous plants for computer imagery purposes. Computer Graphics, 22(4):141-150, 1988. 